\title{
LOKALISIERUNG IM KLISCHEE - ORIENTALISMUS IN GEGENWÄRTIGER POPMUSIK
}

\author{
Markus Wyrwich
}

\begin{abstract}
»Once we begin to think of Orientalism as a kind Western projection onto and will to govern over the Orient, we will encounter few surprises« (Said 2003: 95).
\end{abstract}

\section{Einleitung}

Zwei Palmen und drei Gewürzhäufchen, im Hintergrund ein kleines, in einer Wüste stehendes von der Abendsonne rot eingefärbtes Haus mit einer Kuppel, unterhalb des Bildes in großen eckigen Buchstaben das Wort »Oriental«. In dieser Form ist das Verpackungsmaterial eines Chips-Produktes mit der Geschmacksrichtung »Oriental« gestaltet. »Lassen sie sich verführen«, verheißt die Werbebotschaft, »von der würzig-scharfen Würzmischung. Geheimnisvoll wie der Orient, so schmecken Chipsfrisch Oriental. «1

Dass Lebensmittel generell in komplexen soziokulturellen Bedeutungszusammenhängen stehen können, ist spätestens mit Roland Barthes' Mythen des Alltags (1964) besonders anschaulich verdeutlicht worden. So beschreibt Barthes in seinem Kapitel »Beefsteak und Pommes frites « z.B., wie beide Lebensmittel in bestimmten Kontexten im Frankreich der Mitte des 20. Jahrhunderts »nationalen Glanz « vermitteln und eine patriotische Haltung ausdrücken sollten (Barthes 1964: 37f.).

Vor diesem Hintergrund stellt sich die Frage, wie das Konsumieren der als exotisch angepriesenen »Chipsfrisch Oriental« kulturell gedeutet werden kann. Für die offensichtliche Primärzielgruppe, die westlichen Konsumenten, versprechen die Chips, geschmacklich den Orient zu repräsentieren, indem sie etwas würziger, schärfer und damit wohl aufregender zu sein vor-

1 Das Lebensmittelprodukt »Chipsfrisch Oriental« wird hergestellt von der Kölner Snack und Gebäck GmbH. Der zitierte Werbeslogan ist auf der Webseite des Herstellers angeführt: http://www.funny-frisch.de (Stand vom 3.3.2006). 
geben, als das, was normalerweise verspeist wird. Die Hauptgrundlage des Snacks sind allerdings dünn geschnittene frittierte Kartoffelscheiben, eine originär westliche Erfindung mit Ursprüngen in den USA im 19. Jahrhundert, auf die das vermeintlich orientalische Gewürz lediglich gestreut ist. Die Struktur der Chips ist dem westlichen Konsumenten vertraut; sie hält das exotische Ausmaß des Gesamtproduktes domestizierend in Grenzen. Es liegt eine unbewusst ausgeführte postkolonialistische Handlung und genüssliche Akzeptanz von Inkorporation und Appropriation einer andersartig wahrgenommenen Kultur vor.

Diese auf den ersten Blick etwas radikal anmutende These lässt sich in aller Ausführlichkeit untermauern, insbesondere mit Hilfe von Edward Saids Studie Orientalism von 1978, die nach ihrem Erscheinen in allen kulturwissenschaftlichen Disziplinen nachhaltige Beachtung fand und auch für diesen Aufsatz methodisch wie inhaltlich eine tragende Rolle spielt. Ähnlich den Chips, diesem durch und durch westlichen Produkt, das sich durch ein paar Körnchen Gewürz in ein Symbol des Orients verwandelt und diesen goutierbar macht, werden auch Produkte der Musikindustrie durch vergleichbare Prozesse des Würzens für die Konsumenten neu verortet. Am Beispiel der Titel »Buttons« von den Pussycat Dolls und »Shiver« von Maroon 5 sollen diese Prozesse deutlich gemacht werden.

\section{Orientalismus}

Unmittelbar auf die erstmalige Publikation von Saids Orientalism folgten mehr als sechzig kontroverse Reaktionen allein in US-amerikanischen und britischen Fachzeitschriften (vgl. Macfie 2000: 179). Saids Thesen und methodische Ansätze beeinflussten nach und nach verschiedene kulturwissenschaftliche Fachrichtungen und brachten lebhafte Debatten zu Gegenständen hervor, denen zumeist entweder durch Orientalism überhaupt erst Aufmerksamkeit zuteil wurde oder die nach zahlreichen vorausgegangenen Analysen nun unter neuen Blickwinkeln problematisiert wurden. So reihen sich etwa in der Filmwissenschaft Ende der 1980er Jahre eine Vielzahl von Arbeiten aneinander, die sich mit Darstellungen vermeintlich arabischer Kultur und Ethnizität in US-amerikanischen Filmen auseinandersetzen (vgl. z.B. Browne 1989; Georgakas 1989; Michalek 1989; Shaheen 1987). In ähnlicher Weise akkumulieren sich Anfang der 1990er Jahre in der klassisch ausgerichteten Musikwissenschaft Aufsätze zu Opern wie beispielsweise Aida, Carmen oder Samson et Dalila, allesamt Werke, die nun erstmalig mit klarem Fokus auf ihre orientalistischen Komponierweisen hin untersucht wer- 
den (vgl. Locke 1991; McClary 1992; Robinson 1993). Im Jahre 1998 publizierte Derek Scott eine hervorragend recherchierte musikwissenschaftliche Untersuchung, die eine umfassende Liste orientalistischer Stilmittel hervorbrachte (vgl. Scott 1998). Auch wenn sich seine Analysen ausschließlich auf Werke der Kunstmusik beschränken, können seine Ergebnisse in vielen Fällen ebenso für Untersuchungen von Popmusikkompositionen herangezogen werden. Fragen zu Sounddesign, Album-Cover, Inhalten von Pressemappen oder zu audiovisuellen Darstellungsformen werden in Scotts Ansatz erwartungsgemäß nicht tangiert. Innerhalb der Forschung zur populären Musik, wo solche Fragen eine hohe Relevanz besitzen, hat sich bis dato, abgesehen von einem Artikel von Olivia Bloechl (2005) zu Stings »Desert Rose«, auch noch keine ausgiebige Auseinandersetzung mit Orientalismus und Orientalism abgezeichnet, obwohl die aktuelle Relevanz und Brisanz der Thematik auf der Hand liegt.

Analog zum Verständnis Saids und anderer Autoren (vgl. Browne 1989; Locke 1991; McClary 1992), wird in dieser Arbeit »Orientalismus « als eine bewusst oder unbewusst praktizierte Methode angesehen, aus explizit westlicher Perspektive »orientalische « Kulturen, Landschaften, Menschen und Völker zu repräsentieren bzw. darzustellen. Die geographischen Grenzen eines so genannten »Orients« sind dabei auffällig diffus, sodass Länder z.B. aus dem islamischen mittleren Osten sowie Ost- und Südasien mit ihren jeweiligen in sich bereits höchst komplexen Kulturen in orientalistischen Erzeugnissen häufig pauschal in ein und derselben Kategorie vorgefunden werden können.

\section{Der Orientalismus-Trend in der gegenwärtigen Popmusik}

Seit Ende der 1990er Jahre lässt sich eine signifikante Anhäufung orientalistischer Popsongs quer durch alle Veröffentlichungen von Major-Companys der westlichen Musikindustrie feststellen. ${ }^{2}$ Insbesondere international erfolgreiche Künstler und Acts, die dem weit gefassten Marktsegment »HipHop« zugerechnet werden können, haben einen wesentlichen Anteil an

2 Eine kleine Auswahl international erfolgreicher Songs - neben den in der Arbeit weiter unten genannten: Sean Paul: »Gimme The Light« (2002), Holly Valance: »Kiss, Kiss« (2002), Beyoncé: »Baby Boy« (2003), 50 Cent: »Candy Shop« (2005), Eminem: »Ass Like That (2005), Pussycat Dolls: »Don't Cha« (2005), Chemical Brothers: Push The Button (2005), Shakira: »Hips Don't Lie« (2006), Rihanna: »Unfaithful« (2006), Sugababes: »Easy« (2006), Justin Timberlake: »What Goes Around« (2007), Shakira \& Beyoncé: »Beautiful Liar« (2007). 
dieser Entwicklung. Als hervorstechende, relativ frühe Beispiele können Missy Elliots »Get Ur Freak On« (2001) oder Truth Hurts' kommerziell höchst erfolgreicher Song »Addictive« (2002) angeführt wurde. Der letztgenannte Song wurde von Dr. Dre produziert, der mehrere Bollywood-Gesangssamples verwendete sowie einen Beat mit Tabla-, Clap-, und Cymbal-Sounds programmierte.

Laut dem Musikjournalisten Kevin Braddock sei in erster Linie der »Hip Hop Underground « als essentieller Einflussfaktor für international renommierte HipHop Produzenten anzusehen. Konkret seien vor der globalen Ausbreitung zunächst lokal, ungeachtet jeglicher rechtlichen Restriktionen, diverse »Bollywood Samples « kursiert und zwar »in the depths of New York's hiphop underground« (Braddock 2005). Für diese Entwicklung führt der Autor eine sehr pragmatische Begründung an: »Principally, sample sources for contemporary hip hop and R\&B - James Brown, Funkadelic and Rick James - have long since run dry« (ebd.). Demzufolge mussten schlicht neue Sample-Quellen erschlossen werden, die z.B. in den Musiken der Zuwanderer in westliche Metropolen gefunden wurden. Für Braddock ist in diesem Zusammenhang neben New York besonders der westliche Teil Londons mit seinem relativ hohen Anteil indischer Immigranten hervorzuheben, der der Sampling-Szene unverbrauchtes Material bescherte. So soll z.B. der international erfolgreiche Produzent Tim »Timbaland«Mosley allein an einem Tag mehr als 3000 britische Pfund für Indische desi und bhangra CDs ausgeben haben (ebd.). Für Braddock steht fest, dass die internationale HipHop-Szene unmittelbar den gegenwärtigen Orientalismus-Trend für die gesamte Popmusik induzierte, »because where hip hop producers now lead, pop inevitably follows « (ebd.).

Diese These wirft jedoch einige Probleme auf. Zunächst ist ein Unterscheidungsversuch zwischen HipHop und Pop bei transnational ausgewerteten Musikphänomenen nur durch ideologisch stark aufgeladene Kategorien aufrechtzuerhalten; vielmehr muss hier von komplexen Interrelationen die Rede sein, was beispielsweise an dem orientalistischen Werk »I'm A Slave 4 $U_{\ll}$ (2001) deutlich wird: interpretiert von der Popsängerin Britney Spears und komponiert sowie produziert vom HipHop-Act Neptunes. Was jedoch Braddocks These entscheidend in Frage stellt, ist die Tatsache, dass kurz vor und parallel zu den Entwicklungen im HipHop verschiedene orientalistische Phänomene bereits in anderen populären Musikgenres auftauchten. Ein prominentes Beispiel ist »Desert Rose « aus dem Jahre 1999, ein Kollaborationsprojekt von Sting und dem algerischen Sänger Cheb Mami. Des Weiteren ist seit Ende der 1990er Jahre ein signifikanter Zuwachs von orientalisch anmutenden CD-Kompilationen mit Titeln wie Arabian Rhythms oder Arabic 
Groove auf dem westlichen Musikmarkt zu konstatieren (vgl. Bloechl 2005: 134). Diese Beobachtungen lassen darauf schließen, dass der OrientalismusTrend zum einen eine genreübergreifende Qualität hat und zum anderen wesentlich durch außermusikalische Faktoren bedingt ist. Diese Schlussfolgerung zieht auch Oliva Bloechl. Sie verweist auf zahlreiche in den Medien im hohen Maße thematisierte Konfliktereignisse zwischen orientalischen und okzidentalen Ländern und Interessensgruppen. Neben dem Golf Krieg von 1991, der US-amerikanischen Irak-Invasion im Jahr 2003 und dem seit vielen Jahren schwelenden Israel/Palästina-Konflikt hebt sie dabei besonders die nachhaltige Wirkung der Terroranschläge vom 11. September 2001 für das belastete Verhältnis hervor (vgl. ebd.: 133f.). In Analogie zu Edward Saids Thesen betont sie in diesem Kontext die unmittelbaren Verflechtungen von politischen Motivationen und kulturellen Bedürfnissen, die bei solchen Konflikten stets eine Rolle spielen. Unter Bezugnahme auf eine historische Studie über Tourismus (vgl. Lew/Hall/Williams 2004) schreibt Bloechl:

»European and American colonial history from 1500 onward has demonstrated a direct relationship between state-sponsored violence and the development of consumers' taste for products or behaviours of a violated people« (Bloechl 2005: 134).

Anschließend verknüpft sie die »Orient/Okzident-Krise« direkt mit dem ansteigenden Verkauf orientalistischer Musikprodukte auf dem westlichen Musikmarkt seit Ende der 1990er Jahre und charakterisiert diese Entwicklungen als »lebendigen Orientalismus «:

»British or American consumers' desire to possess cultural artifacts associated with the Middle East must be [...] contextualized as part of the living history of Orientalism« (ebd.).

Der Unterschied zwischen Bloechls und Braddocks Herangehensweise bei der Analyse des Trends ist evident. Während Bloechl in ihre Arbeit keine musikindustrie- bzw. musikgenrebezogenen Überlegungen mit einfließen lässt, konzentriert sich Braddock vornehmlich auf eben solche und wehrt sich sogar vehement gegen eine Reflektion etwaiger außermusikalischer Einflussfaktoren: »It may ultimately be pointless attaching any kind of Orientalist dialetic to pop music. Unlike literature or conceptual art, pop music is meant to be danced to rather than deconstructed. It really is that simple (Braddock 2005).

Ganz so einfach ist es sicher nicht, was durch die Breite an Erklärungsversuchen in den sich zum Teil inhaltlich diametral gegenüberstehenden Artikeln ersichtlich wird. Doch gerade die Kombination der Argumentationen 
beider Artikel ist vielversprechend, um ein erstes Verständnis des Orientalismus-Trends gewinnen zu können; eine Reihe von Ergänzungen sind jedoch notwendig: Laut Simon Frith (2001: 48) hätten Plattenfirmen seit jeher miteinander im Wettbewerb gestanden, indem sie eher erfolgreiche Produkte eines Konkurrenten kopiert hätten, als eigenständig in etwas völlig Anderes zu investieren. Dieser Sachverhalt lässt sich bereits in den 1940er Jahren während der Herausbildung des oligopolistischen Musikmarktes in den USA nachvollziehen, wie David Berger und Richard Peterson (1975: 163) zeigen konnten. Zudem muss berücksichtigt werden, dass Musikschaffende wie Komponisten und Produzenten immer von strukturellen und ästhetischen Diskursen beeinflusst sind, die vereinfacht in drei Kategorien unterteilt werden können: Insbesondere bei Singleproduktionen von Major-Plattenfirmen für den internationalen Musikmarkt herrscht auf einer ersten Ebene ein recht stabiler Diskurs über Struktur, Länge, Produktionsqualität, thematische Gestaltung, Instrumentierung und weitere grundsätzliche Kriterien eines Songs vor. Von Diskursen zweiter Ebene kann im Bezug auf Trends gesprochen werden, die bewusst oder unbewusst von Musikern, Produzenten, Komponisten oder A\&R-Managern aufgenommen werden und damit essentiellen Einfluss auf musikalische Konzeptionen haben. Relativ trivial, aber trotzdem erwähnenswert ist der Sachverhalt, dass ein solcher Trend solange anhält, bis er sich entweder zu einer eigenen Genrekategorie etabliert oder bis er von einem neuen abgelöst wird, sofern sich die Verkaufszahlen der durch inn geprägten Musikprodukte ökonomisch unzureichend entwickeln. Nach diesem Verständnis lässt sich noch eine dritte Ebene definieren, die sich auf einen internen Diskurs zur Komposition, Produktion und Performance eines Songs innerhalb eines Teams bestehend aus LabelPersonal, Management, Produzenten und Musikern bezieht und für die Einzigartigkeit eines jeden Produktes entscheidend ist.

Die Durchsetzungsfähigkeit des Orientalismus-Trends muss zunächst grundsätzlich mit diesen musikindustrieimmanenten Erwägungen erklärt werden. Um sich neben Bloechls und Braddocks Erwägungen der Frage noch detaillierter nähern zu können, warum das Phänomen überhaupt auf ein weit reichendes Interesse bei westlichen Rezipienten stößt und sich damit als ein lebendiger Diskurs zweiter Ebene innerhalb der Musikindustrie etablieren konnte, werden im Folgenden zwei orientalistische Musikbeispiele ausführlich analysiert. 


\section{4. »Buttons« von den Pussycat Dolls}

Der Name der US-amerikanischen Popgruppe wird durch die Kombination der allesamt sexuell konnotierten Begriffe »pussy«, »cat« und »dolls« gebildet. »Pussy«, häufig umgangssprachlich als Synonym für Vagina oder zur despektierlichen Bezeichnung einer weiblichen Person gebraucht, steht ganz am Anfang und erhält damit eine besondere Gewichtung. Das Wort »cat« deutet zum einen metaphorisch Sensibilität und Verschmustheit an, gleichzeitig schwingt eine Spur von Selbstbestimmung, Animalität sowie ein gewisser Grad an Gefahr mit, die von dem mit Krallen ausgestatteten Katzentier ausgehen kann. »Dolls« sind in der Regel Spielzeugobjekte, die be- und entkleidet werden können und deren Schicksal ihrem Besitzer überlassen ist. Der Begriff wird häufig mit sexistischem Unterton als Bezeichnung für Frauen gebraucht, die einem brust- und gesäßbetonten schlanken Schönheitsideal entsprechen. Insbesondere in Bezug auf die Strip- und Tanzlokalkultur, mit deren Symbolik die Pussycat Dolls (PCD) vermarktet werden ${ }^{3}$, erhalten alle drei Begriffe eindeutige Konnotationen mit einem sich wechselseitig verstärkenden Einfluss, sodass bereits im Bandnamen eine regelrechte Überfrachtung sexueller bzw. sexistischer Bedeutungsebenen impliziert ist. Durch eine Betrachtung der Gruppenkonstellation und des Auftretens der Bandmitglieder lässt sich dieser Grundeindruck bekräftigen. Während ein ungeschriebenes Gesetz der Musikbranche vorzugeben scheint, dass Boy- oder Girlgroups mit maximal fünf Personen auftreten könnten (man denke an Take That, NSync', Destiny's Child oder an die Spice Girls), bestehen die PCD aus sechs Mitgliedern. Individuelle Charaktereigenschaften, die beispielsweise bei den Spice Girls mit Titulierungen wie »Sporty-« oder »Posh-Spice« (vgl. Leach 2001: 165) beim Rezipienten suggeriert werden sollten, stehen bei den PCD nicht im Vordergrund, sondern werden vielmehr in der Pressemappe des Labels (wo alle Attributszuschreibungen im Plural verfasst sind) oder in Interview-Situationen bewusst negiert. Bei einem Promotionauftritt im Rahmen von TV total (neben Wetten, dass...? Deutschlands relevantester Fernsehsendung für den Musikmarkt ${ }^{4}$ ) konnte

3 In der Bandbeschreibung, entnommen der Pressemappe, herausgegeben von der Plattenfirma A\&M Records, ist von den Ursprüngen der PCD als reiner Tanzformation die Rede: »Founded by choreographer Robin Antin in 1995, the Pussycat Dolls began as a burlesque dance revue based in Los Angeles« (A\&M Records 2005).

4 Aufgrund von Erfahrungswerten hinsichtlich positiver Entwicklungen in den Charts von Acts nach Auftritten in beiden Shows werden TV total und Wetten, dass...? oftmals in einem Atemzug als wichtigste Promotionplattformen im deut- 
nachvollzogen werden, dass diese Imagestrategie von allen Gruppenmitgliedern stark verinnerlicht wurde. Auf die Frage des Moderators Stefan Raab, wer denn die beste Sängerin sei, antworteten alle PCD-Mitglieder plattitüdenhaft beinahe gleichzeitig, dass jede in gleicher Weise talentiert sei und keine besonders hervorstechen würde. ${ }^{5}$ Gleichwohl besteht kein Zweifel daran, dass Nicole Scherzinger in jedem Lied des Debütalbums die Leadvocals singt und auch bei den Backings die hörbar tragende Rolle spielt. Auch die Tatsache, dass Scherzinger laut Album Credits an zwei Songs kompositorisch beteiligt ist, was in vielen Musikerbeschreibungen besonders hervorgehoben wird, um handwerkliche Fähigkeiten und die Glaubwürdigkeit von Musikern zu inszenieren, soll offensichtlich kein vordergründiges Element des PCD-Images sein.

Auf dem Albumcover werden alle Frauen seitlich mit ihren sehr ähnlichen schlanken physikalischen Erscheinungen mit gesäß- und brustbetonenden Posen präsentiert. Abgesehen von einer minimalen Hervorhebung der in der Mitte stehenden Nicole Scherzinger durch einen dezent mystisch wirkenden aufhellenden Bildbearbeitungseffekt um ihre Haare und ihren Bauch herum impliziert das Imagedesign für die PCD keine herausragende körperlichen und persönlichen Diversitäten der Gruppenmitglieder, sondern ist in erster Linie darauf ausgelegt, lediglich mit ethnischen Unterschieden eine sexuelle Reizüberflutung, orientiert an einem westlichen Schönheitsideal, zu produzieren.

Am 26. Juni 2006 wurde der Song »Buttons« unter Mitwirkung des USRappers Snoop Dogg als vierte Single des PCD-Albums veröffentlicht. In den darauf folgenden Wochen und Monaten wurde der kommerzielle Erfolg der Single anhand von hohen Airplay-Zahlen, Top-5 Charts-Platzierungen in allen großen Ländern des westlichen Musikmarktes sowie durch die Auszeichnung "Best Dance Video « bei den MTV Video Music Awards deutlich. ${ }^{6}$

Im Kern besteht »Buttons « aus insgesamt zwölf 8-Takt-Schemata (zur Veranschaulichung der Analyse siehe Abb. 1 auf der folgenden Seite). Das erste Schema (Takt 2-9) ist mit einem Rap von Snoop Dogg ausgestaltet, wo

schen Fernsehen benannt, beispielsweise durch den Musikmanager Peter Cadera in einem Interview mit der ZEIT vom 7.2.2006 (vgl. Ott 2006).

5 Vgl. TV total-Sendung vom 16. November 2005, ausgestrahlt um $22.15 \mathrm{Uhr}$ auf ProSieben.

6 Auch wenn die Zitierwürdigkeit von Wikipedia nach wie vor umstritten ist, bietet dieses interaktive usergenerierte Internetangebot in der Regel sehr verlässliche Informationen in übersichtlicher Form zu diversen Charts-Platzierungen von Künstlern und Bands; so auch im Falle der PCD: http://en.wikipedia.org/ wiki/The_Pussycat_Dolls (Stand vom 26.3.2007). 


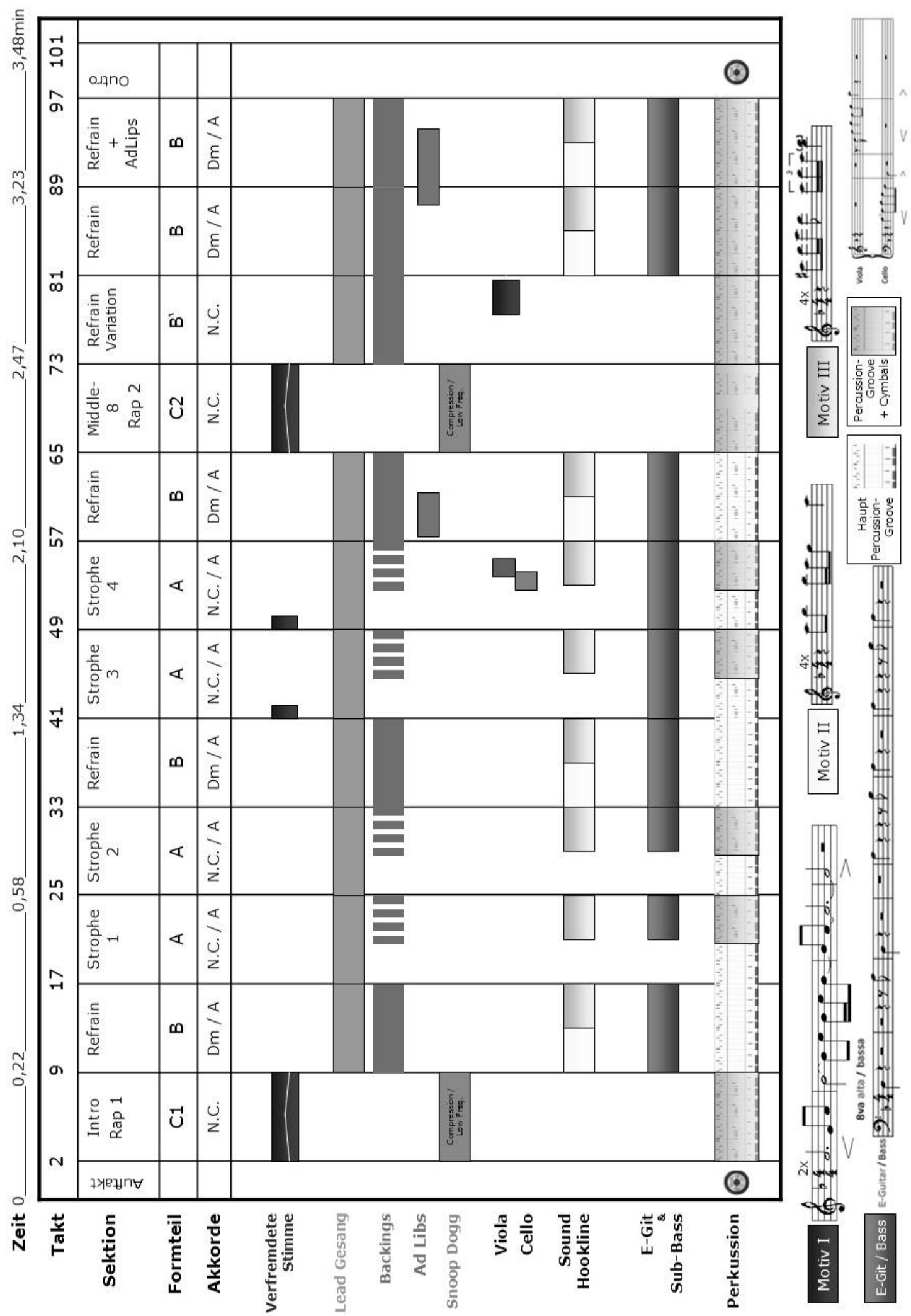

Abb. 1: »Buttons « - Strukturanalyse ${ }^{7}$

7 Vgl. die Graphik in Farbe und mit guter Auflösung unter der URL: http://www. thoughts-and-sounds.com/wissenschaft/aspm_graphik1. 
nach sich unmittelbar ein 8-taktiger Refrain erstmalig anschließt. Im weiteren Verlauf folgen zweimal hintereinander zwei 8-taktige StrophenSchemata plus Refrain, bevor eine Art Middle-8 erneut mit einem Rap aufwartet. Schließlich erklingen noch drei 8-taktige Refrains bzw. Refrainvariationen. Insgesamt folgt der Song damit rein strukturell einem westlichen Popsong-Standard, bei dem der Refrains, der bei »Buttons « in Reinform und mit seinen Variationen mehr als die Hälfte des gesamten Liedes ausmacht, im Vordergrund steht und beim Rezipienten mit seiner Hookline für den entscheidenden Wiedererkennungswert sorgen muss. Dieser ist bei »Buttons « zum Teil auch durch die dominante Rhythmusgruppe gewährleistet. Der durchgehend im 4/4-Takt komponierte und mit einem Tempo von $105 \mathrm{bpm}$ moderat schnelle Song hat einen repetitiven Groove zur Grundlage, mit Betonung durch einen tiefen Kesseltrommelsound (vgl. Abb. 2) auf den Zählzeiten $» 1 \ll$ und $\gg 2+\ll$, wodurch nach westlichen traditionellen Maßstäben ein synkopierter Grundrhythmus vorliegt. Darüber hinaus produziert der Kesseltrommelsound durchgehend den Ton ${ }_{1} D$, der hier die Funktion eines Orgelpunkts bzw. Drone bekommt. Auf den Zählzeiten » 2 « und »4« erklingen hand claps, die bei der Programmierung des Beats - sofern hierbei kein Sample verwendet wurde - aller Wahrscheinlichkeit nach bewusst unquantisiert gelassen wurden, um das Bild eines spontanen, mit rein akustischen Mitteln durchgeführten, sozialrelevanten Musizierens einer kleinen Gemeinschaft latent zu evozieren. Essentiell für den Beat sind zudem mittelhohe, in Achtelfiguren programmierte Kesseltrommelsounds sowie teilweise in Sechzehntelabständen erklingende Percussionsounds (mit einem hervorstechend Triangelklang), die dem Song seine grundlegende Lebendigkeit verleihen.

\begin{tabular}{|c|c|c|c|c|c|c|c|c|c|c|c|c|c|c|c|c|}
\hline $\begin{array}{l}\text { Mittlere } \\
\text { Kessel- } \\
\text { trommel }\end{array}$ & 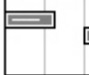 & 回 & & 回 & $\square$ & 回 & & $\square$ & 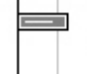 & 回 & & 回 & 回 & 回 & & $\square$ \\
\hline Triangel & \begin{tabular}{|l}
$g$ \\
gedăm
\end{tabular} & pft & 回 & 回 & $\begin{array}{l}\square \\
\text { offen }\end{array}$ & & & & & & & & & & & \\
\hline Claps & & & 甸 & & & & 匍 & & & & 包 & & & & 匐 & \\
\hline (tief) & 1 & + & 2 & + & 3 & + & 4 & + & 1 & + & 2 & + & 3 & + & 4 & + \\
\hline
\end{tabular}

Abb. 2: »Buttons« - Matrix-Editor-Darstellung des grundlegenden Grooves

Bezeichnenderweise finden sich alle hier angeführten Perkussionsinstrumente auf Scotts Liste wieder (Scott 1998: 327), was darauf hindeutet, dass auch die Produktion von »Buttons « durch eine weit verbreitete orientalistische Sichtweise beeinflusst war, orientalische Musik sei zwangsläufig rhythmisch sehr lebendig und werde stets mit unelektrifizierten Instrumenten 
ausgeübt, womit sich unterschwellig ideologisch hochbrisant eine von Said mehrfach nachgewiesene Vorstellung verbindet, der Orient an sich sei ursprünglicher, leidenschaftlicher, spontaner und damit weniger zivilisiert und minder rational als der Okzident (vgl. Said 2003: 49).

»Buttons« beinhaltet harmonisch lediglich die Akkorde d-Moll und A-Dur und ist ausschließlich mit modalen Skalen komponiert. Der Song weist insgesamt drei wichtige Motive auf, wovon die ersten beiden um den Zentralton $a$ und das dritte Motiv um den Zentralton $d$ kreisen.

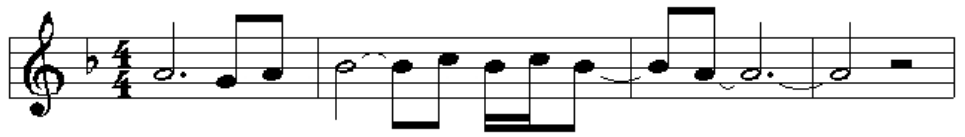

Abb. 3: »Buttons« - Motiv I

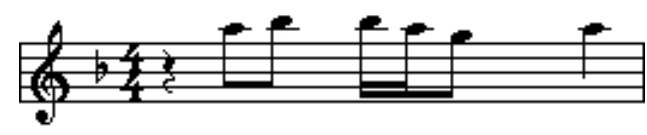

Abb. 4: »Buttons $\ll-$ Motiv II

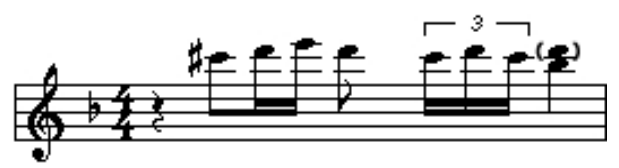

Abb. 5: »Buttons $«-$ Motiv III

Alle Motive bewegen sich in Halb- und Ganztonschritten, zum Teil relativ schnell in Sechzehnteln. Zudem sticht in Motiv III eine Triole hervor, die zur rhythmischen Komplexität bzw. Schnelligkeit der Melodie beiträgt. Motiv III erhält u.a. dadurch eine exponierte Stellung im Song, als es insgesamt 36 Mal auftritt. Diese Beobachtungen zur Melodiebildung lassen darauf schließen, dass sich die Komponisten des Songs gängiger orientalistischer Stilmittel bedient haben, die auch in zahlreichen Beispielen der Kunstmusik von Scott zusammengetragen werden konnten (vgl. Scott 1998: 308f.).

In der Instrumentierung des Stücks dominieren die Percussions. Zusätzlich zu den oben benannten rhythmischen Elementen, die den grundlegenden Groove erzeugen, erklingt ganz am Anfang und am Ende deutlich hörbar ein Gong, der »Buttons « eine mystische Note verleiht, indem er inn wie eine Traumsequenz ein- und ausleitet. ${ }^{8}$ Neben einem tiefen unisono gespielten

8 Ursprünglich sind Gongs dem südost-asiatischen Kulturraum zuzuordnen, fanden aber bereits im 18. Jh. Einzug in die westliche Kunstmusik (vgl. Blades/ Holland/Thrasher 2001). Selbst wenn Gongklänge für westliche Rezipienten mittlerweile nicht mehr allzu ungewöhnlich sein dürften, hat sich ihr »exotisches Moment « nicht völlig neutralisiert. Nach wie vor werden sie häufig etwa 
Klangkonglomerat von Sub-Bass und E-Gitarren, das sich vor allem in Discotheken voll entfalten kann, sind in erster Linie vermeintlich akustische Instrumente (bzw. hochqualitative Samples oder Synthesizerprogramme) im Vordergrund; dementsprechend ist ein kleines Zwischenspiel zwischen Takt 53-56 mit Viola und Cello arrangiert. Des Weiteren sticht bei der Instrumentierung von »Buttons« ein charakteristischer Sound hervor, der durch das mehrfache Spielen der Motive II und III die Funktion einer Soundhookline ${ }^{9}$ erhält. Aller Wahrscheinlichkeit nach wurde er ursprünglich durch eine Überlagerung verschiedener Streicher-Sounds mit leicht unterschiedlichen Tonhöhen kreiert, die bewusst eine nicht-»wohltemperierte « Stimmung erzeugen. Die Klangfarbe des synthetischen Sounds ermöglicht nicht nur Assoziationen etwa mit Violine und Cello, sondern durch das leicht Metallische auch mit einer Sitar. Damit hat der Sound insgesamt eine vielschichtige Qualität, die mehrere mit dem Orient aus westlicher Sicht pauschal in Verbindung gebrachte Instrumente gleichzeitig anzudeuten vermag.

Neben dem gradlinigen 8-taktigen Periodenbau sticht besonders die Produktionsqualität als ein typisch westliches Merkmal heraus. Alle Stimmen und Instrumente sind komprimiert und mit Equalizern bearbeitet, sodass ein frequenztechnisch gut sortiertes, im Sinne der Gesangsstimme und Hookline hierarchisiertes Mixing-Resultat vorliegt. Dieses ist nach grundlegenden ästhetischen Konventionen ausgerichtet, die im Hinblick auf Radio- und TVAirplay kaum umgangen werden können. Des Weiteren gewährleistet das Mixing die multiple Einsatzmöglichkeit des Songs, sei es in Großraumdiscotheken mit extrem basslastigen Beschallungssystemen oder in privaten Haushalten mit kleinen Küchenradios. Alle »exotischen« Elemente des Songs sind quasi dieser ästhetischen Konvention untergeordnet; sie gewährleistet gemeinsam mit dem 8-taktigen Periodenbau die Dekodierbarkeit des Songs für westliche Rezipienten nach ihren Hörgewohnheiten.

Im Kern des Liedtextes von »Buttons « steht das Verhältnis zweier Personen, welches durch polarisierende Gefühlszustände wie Verlangen und Abweisung beschrieben ist. Der Ich-Erzähler kämpft leidenschaftlich mit of-

bei Hörspiel- oder Filmproduktionen als Sound-Effekt verwendet, um Mysthisches oder Surreales ein- und auszuleiten.

9 Die Einführung der Begrifflichkeit "Soundhookline« erfährt durch zahlreiche Popmusikbeispiele ihre Berechtigung: Neben Hooklines, also eingängigen Refrainmelodien, tauchen in vielen Songs zusätzliche repetitive Elemente auf, die ebenfalls durch eingängige Melodien hervorstechen, jedoch explizit in unmittelbarer und unzertrennlicher Verbindung mit einem sehr charakteristischen Sound stehen. Zwei anschauliche Soundhookline-Beispiele sind das kurze Gitarrenlick am Ende eines jeden Refrains in Britney Spears' »Toxic « (2003) oder das weit nach vorn gemischte Flötenspiel in Jennifer Lopez' »Jenny From The Block« (2002). 
fensiv artikulierten sexuellen Angeboten (»I'm liking the physical [...] I'm a sexy mama [...] I'm about to blow«) um die Gunst des Gegenübers, dessen Reaktionen jedoch als kühl und distanziert beschrieben werden: »But you keep fronting [...] But I ain't seen nothing «. Sofern man einwenden möchte, dass selbst Muttersprachler nicht zwingend einem Musiktext die volle Aufmerksamkeit schenken - wie es Simon Frith in einer Studie nachweisen konnte (vgl. Frith 1988: 119f.) -, kann beim vorliegenden Beispiel darauf verwiesen werden, dass die gesanglichen Interpretationen der im Text verankerten binären Rollenaufteilung voll und ganz entsprechen: Die PCD, allen voran Nicole Scherzinger, singen intensiv und hingebungsvoll, zum Teil mit für musikalischen Orientalismus typischen Melismen, wohingegen Snoop Dogg seinen typischen Rapstil bedient, der hier als Gegenpol Gelassenheit, Souveränität, Stärke und Maskulinität ausdrücken soll. Bemerkenswerterweise findet sich diese stets sexuell konnotierte, stark klischeebehaftete Genderunterscheidung in vielen anderen Quellen direkt übertragen auf den Unterschied von Okzident und Orient wieder. Es liegen unzählige westliche Erzählungen und Phantasien vor, die den Orient zum einen direkt mit »excessive freedom of intercourse «, »perversion« oder »dangerous sex« (Said 2003: 167) in Verbindung bringen und inn zum anderen durchweg mit vorgeblich weiblichen Attributen gleichsetzen: »The Orient was routinely described as feminine, its riches as fertile, its main symbols the sensual woman, the harem and the despotic « (Said 1985: 103).

Auch im Video zu »Buttons« taucht diese grundlegende binäre Konstruktion auf. Bereits die Anfangsszenen inszenieren den Rapper »cool«, fest an einem Punkt stehend, mit dezent eingesetzten Körperbewegungen und Gesten durch teilweise von unten aufgenommene Bilder, die ihn deutlich groß erscheinen lassen. ${ }^{10}$ Die Annäherung zunächst nur von Scherzinger und im weiteren Verlauf von allen PCD im aufreizenden Gleichschritt vermittelt den Eindruck, als ob sich alle Frauen dem einzigen im Video auftretenden Mann für sexuelle Dienste anbieten würden. Diese Konstellation muss als Reproduktion einer explizit westlichen Haremsphantasie verstanden werden, da im orientalischen Kulturraum Haremsmythologien und -vorstellungen marginal oder gar nicht sexuell konnotiert sind (vgl. Mernissi 2001).

Der überwiegende Teil des Videos ist mit Tanzeinlagen und Requisiten konzipiert, die zum einen an Strip- und Tanzlokaldarbietungen erinnern wie z.B. Stretch-Bewegungen an einer langen Ballettstange und diverse

10 Bei dieser Inszenierung von Snoop Dogg wird man schnell an Richard Dyers These erinnert, dass viele Darstellungsformen von Männlichkeit in der Popularkultur einen »phallischen Mystizismus« bedienen und reproduzieren (Dyer 1992: 103-119). 
Striptease-Andeutungen - und sich zum anderen ästhetisch eindeutig an Bollywood-Filme anlehnen. Letzteres wird an den Tanzchoreographien deutlich, die aus von oben gefilmten kreisförmigen Bewegungsmustern bestehen oder sich an bildlichen Darstellungen von Hindu-Gottheiten wie Vishnu, Kali, Brahma oder Shiva orientieren. ${ }^{11}$ Darüber hinaus weist die farbliche Gestaltung mit einem hervorstechenden, sanft dunklen Sonnenlichtgelb sowie der Einsatz eines großen Scheinwerfers zur Imitation einer auf- oder untergehenden Sonne auf den Versuch hin, vermeintlich orientalische Naturbezüge herzustellen bzw. eben solche aus Bollywoodfilmen nachzuahmen. Zudem fällt ein aus Glas- oder Plastikperlen bestehender Glitzervorhang, durch den sich Scherzinger lasziv räkelt, in mehreren Sequenzen des Videos auf. Gerade solche Requisiten sind laut Clare Parfitt (2005: 107) in zahlreichen westlichen Filmen vorzufinden, in denen das pompöse Element zahlreicher Bollywood-Produktionen nachgeahmt bzw. zitiert werden soll. Abschließend ist noch ein im letzten Drittel des Videos zum Einsatz kommender digitaler Effekt zu erwähnen. Dieser erweckt den Eindruck, als würden, durch die Tanzbewegungen der PCD ausgelöst, explosionsartig Flammen den Boden entzünden, ohne dass die Tänzerinnen selbst dadurch gestört werden, ihre Bewegungen fortzusetzen. Verschiedene Vermutungen über das direkte und unterschwellige Wirkpotential dieser Feuerszenen bieten sich an. Es ist nicht auszuschließen, dass vor dem Hintergrund der orientalistischen Musikklänge solche Explosionsbilder bei manchen Betrachtern Assoziationen mit islamistisch motivierten Terroranschlägen hervorrufen können. In erster Linie symbolisieren die Flammen jedoch in verspielter Weise ein ähnliches Gefahrenpotential, wie es mit den Katzenkonnotationen im Act-Namen bereits verankert ist.

Interessanterweise liegen zwei Video-Versionen von »Buttons« vor, eine mit und eine ohne den digitalen Feuereffekt. Dies lässt den Schluss zu, dass die Plattenfirma den Einsatz des Effektes als kontrovers und potentiell problematisch ansah, dabei aber einerseits eine der Promotion von »Buttons « zuträgliche Kontroverse in Kauf nahm (die indes bis dato ausblieb) und

11 Bei diesen Szenen, erstmalig ab 0'40" des Videos, schreitet Nicole Scherzinger mit beschwörerischem, starr in die Kamera gerichteten Blick in einer schwarzen Röhre aufrecht voran; ihr folgen links und rechts jeweils zwei Frauen, die seitlich die innere Röhrenform mit geschmeidigen Armbewegungen betasten. Die konkreten Inspirationsquellen für diese Choreographien sind in BollywoodFilmen zu suchen, die wiederum von klassischen indischen Tanzformen beeinflusst sind, welche laut Judith Hanna durch zahlreiche religiöse Anspielungen geprägt sind: »undergirded by many different religious belief systems and sectarian strands, each with doctrinal [...] and ritual peculiarities [with] a hundred delicate shadings not always obvious to the Western observer « (Hanna 1993: 121). 
andererseits mithilfe der »feuerfreien « Version das Risiko vermied, von Video-Playlisten einzelner Sender ausgeschlossen zu werden, denen die Feuerszenen zu heikel erscheinen könnten, besonders an Tagen mit weiteren Terroranschlägen in westlichen Metropolen. ${ }^{12}$

Zusammengefasst lassen sich auffällige Korrelationen zwischen dem Gesamtprodukt PCD und verbreiteten orientalistischen Vorstellungen konstatieren. Zahlreiche Elemente des PCD-Images sind adäquat positioniert, um besonders im Kontext von »Buttons « eine explizit westliche Haremsphantasie bedienen zu können. Zudem ist die Gesamtästhetik von »Buttons in Form von Musik und Video größtenteils dahingehend ausgerichtet, eine Ursprünglichkeit, Leidenschaftlichkeit, Naturverbundenheit sowie tendenzielle Gefährlichkeit zu inszenieren - alles bekannte Stereotypen, die dem Orient durch Orientalismus im westlichen Kulturraum in jahrhundertealter Tradition nach wie vor zugeschrieben werden (vgl. Said 2003: 49).

\section{5. »Shiver $\ll$ von Maroon 5}

Die US-amerikanische Rockband Maroon 5 veröffentlichte 2002 das Album Songs About Jane. Darin enthalten ist "Shiver «, ein Song der zunächst nicht als Single ausgekoppelt wurde, aber drei Jahre später für die DVD und das Live-Album Live-Friday The 13th als »Opener « ausgewählt wurde und bei zahlreichen Promotionauftritten gespielt wurde. »Shiver « ist aufgrund seiner Struktur mit Vers- und Refrainaufteilungen in Form von 8-taktigen Perioden sowie durch eine Instrumentierung mit Gesang, zwei E-Gitarren, E-Bass und Schlagzeug als ein typisches Rockstück zu bezeichnen. Als rhythmische Grundlage erklingt durchgehend ein hart gespielter Rockbeat im 4/4-Takt bei 86 bpm. E-Bass, Rhythmusgitarre und Sänger intonieren ebenso in einer nach westlichen rockästhetischen Hörgewohnheiten nicht untypischen Art und Weise. Allein ein Gitarrenlick sticht vor dem Hintergrund dieser Ästhetik deutlich hervor:

12 Zumindest auf der in Deutschland vertriebenen Single »Buttons« ist die Version mit Feuer enthalten. Auf der Video-Plattform YouTube sind die meistgeklickten Video-Uploads von »Buttons « jedoch komplett »feuerfrei« (vgl. http://www. youtube.com/results?search_query=buttons+pussycat+dolls; Stand: März 2007), was darauf hindeuten könnte, dass diese Version für YouTube-»Uploader « leichter verfügbar war und sie damit aller Wahrscheinlichkeit nach in den USA am meisten durch die Plattenfirma bzw. entsprechende Medienanstalten verbreitet wurde. 

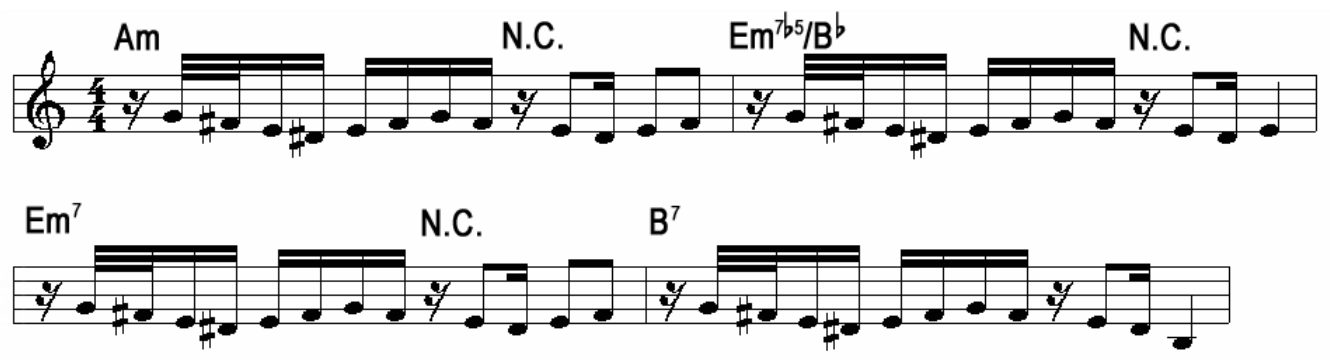

Abb. 6: »Shiver« - Gitarrenlick

Das Lick ist rhythmisch komplex, mit hauptsächlich 16tel- und zum Teil 32stelnoten relativ schnell und schreitet nahezu ausschließlich in Halb- und Ganztonschritten voran. Neben diesen ersten Indizien für eine orientalistische Komponierweise ist es aber im Wesentlichen das Sounddesign, welches für eine »exotische« Dimension des Gitarrenlicks sorgt. Hierfür wurden im Overdub-Verfahren zwei leicht verzerrte E-Gitarren miteinander vermischt, die zwar unisono spielen, aber durch minimale Tonhöhenunterschiede die Norm der temperierten Stimmung negieren; sie erzeugen einen Klang, der von westlichen Rezipienten mit einem weiten Feld orientalischer Instrumente (von der Sitar bis zum Blasinstrument Kaval) assoziiert werden kann. Da das Gitarrrenlick recht standardisiert durch Schlagzeug, E-Bass und Rhythmusgitarre begleitet wird, eröffnet sich nach westlichen rockästhetischen Kriterien ein Spannungsfeld zwischen »Exotik« und »Norm«. Es lässt sich argumentieren, dass das »Exotische « durch sein rhythmisches und melodiöses Gepräge etwas Verspieltes, Mysteriöses, leicht Fragiles verkörpert und von der »Norm«, die für das Ernste, Bekannte, Souveräne und Starke steht, ${ }^{13}$ innerhalb von vier 4/4-Takten domestiziert wird.

Eine solche bipolare Aufteilung lässt sich auch in den Textzeilen von »Shiver« feststellen. Im Kern des Textes thematisiert das lyrische Ich eine Konfliktsituation mit einem weiblichen Gegenüber (»You build me up, you knock me down [...], you are the queen of runaround «), die er mit Worten umschreibt, die die weibliche Person in den Bereich des Magischen, Mystischen und potentiell Gefährlichen rückt: »Immobilized by the thought of you; paralyzed by the sight of you; hypnotized by the words you say«. Diese Wortwahl veranschaulicht besonders deutlich, wie der Orientalismus in der Popmusik neben klanglichen und visuellen Erscheinungsformen auch in Liedtexte Einzug erhält.

13 Diese Attributzuschreibungen sind konstant in orientalistischen Werken immer wieder aufzuspüren. Westliche Menschen seien darin als »rational, peaceful, liberal, logical, capable of holding real values, without supicion« beschrieben, während orientalische Menschen genau gegenteilig porträtiert würden (Said 2003: 49). 
Analog zum Beispiel der Gruppe Pussycat Dolls bleibt die bipolar angelegte inhaltliche Konstruktion in "Shiver « auch bei einer Rezeption ohne besondere Beachtung des Textinhaltes erhalten. Durch den Gesamtkontext der Gruppe Maroon 5 mit ihren ausschließlich männlichen Mitgliedern und einem Albumtitel mit direktem Bezug zu einer weiblichen Person (Songs About Jane) sowie dem Video zur höchst erfolgreichen Single »This Love « mit Bettszenen des Frontsängers und einem Modell, sind viele Voraussetzungen dafür geschaffen, den Inhalt von »Shiver « mit einer heterosexuellen zwischenmenschlichen Beziehung zu assoziieren. Unter Berücksichtung dieses komplexeren Bandkontextes ist die Schlussfolgerung möglich, dass dem orientalistischen Gitarrenlick die Funktion zukommt, die paralysierenden und hypnotisierenden Charaktereigenschaften des weiblichen verspielten, unrationalen Subjekts klanglich auszudrücken. Somit können orientalistische Musikstilmittel also auch zur Konstruktion und Reproduktion geschlechtsspezifischer Vorstellungen verwendet werden, die den Orient ganz konkret mit weiblichen Konnotationen besetzen und die zur Verbreitung von zum Teil eklatant sexistischen Klischees beitragen.

\section{Schlussfolgerungen}

Die Veröffentlichungen und fortwährenden medialen Auswertungen von »Buttons«, »Shiver « und anderen orientalistischen Popmusikerzeugnissen koinzidieren mit einer anhaltenden kulturellen und politischen Spannung zwischen okzidentalen und orientalischen Ländern und Interessensgruppen. Die beiden in dieser Arbeit analysierten Songs tauchen nach wie vor sehr häufig in verschiedenen Medien auf, die tagtäglich in ihren Nachrichtensendungen und Dokumentationen über global wahrgenommene Ereignisse wie den Irakkrieg, den Palästina-Israel-Konflikt, den Iran-Atomstreit oder über Terroranschläge berichten. Auch wenn das jeweilige Gesamtproduktdesign für die Band Maroon 5 und besonders für die PCD für sich stehend viele Kriterien erfüllt, um auf dem westlichen Markt der Kulturindustrie generell erfolgreich sein zu können (etwa mit einer Anhäufung sexueller Komponenten oder einem hohen Kompositions- und Produktionsstandard), besteht ein Zusammenhang zwischen dem Erfolg von Songs wie »Buttons« und »Shiver« und der gegenwärtigen globalen politisch-kulturellen Konfliktsituation. Neben den oben dargestellten musikindustrieimmanenten Bedingungen, die zur Produktion orientalistischer Popsongs führen, sind komplexere Erörterungen zur Ursachenforschung des Orientalismus-Trends nötig. 
»Orientalism serves to control and domesticate [the] threat that Islamic culture represents to Western Europe « (Bernstein/Studlar 1997: 3), so lautet ein vordergründiges Untersuchungsergebnis der Studien Saids und nachfolgender Forscher, das auch im Bezug auf »Buttons« und »Shiver« Gültigkeit besitzt. Beide Songs sind nach formgebenden westlichen Standards kreiert worden, insbesondere in punkto Struktur und Produktionsästhetik, durch die das »Exotische« für westliche Rezipienten in dekodierbarer Form »kontrolliert« und »gezähmt « aufbereitet vorliegt. Darüber hinaus wird durch binäre Konstruktionen vermeintlich Orientalisches als etwas Fragileres und Schwächeres im Vergleich zu westlichen Normvorstellungen dargestellt, was als ein Akt der Domestizierung und Herabstellung des Anderen bewertet werden muss. Die essentielle Frage, warum überhaupt ein Bedürfnis besteht, die vermeintliche Kultur des »Feindes « zu inkorporieren und diese der eigenen unterzuordnen, lässt sich mit psychoanalytischen Thesen näher problematisieren. Laut Frantz Fanon gilt: »When it encounters resistance from the other, self-consciousness undergoes the experience of desire (Fanon 1986: 112). Ein politischer und kultureller westlicher Paternalismus gegenüber orientalischen Ländern oder etwa eine Invasion nicht-islamischer Soldaten in Länder wie Irak oder Afghanistan sind Aktivitäten, die zwangsläufig auf Widerstand stoßen, am spürbarsten in Form von Terror-Anschlägen, welche die Gewaltspirale weiter anheizen. Solange das »orientalische Gegenüber « nicht gänzlich unter Kontrolle gebracht werden kann, (was auch nicht wünschenswert ist, da dies mit militärischen Eingriffen unvorstellbaren Ausmaßes einhergehen würde), bleibt gleichzeitig ein Verlangen nach Kontrolle bestehen, was nach psychoanalytischen Erkenntnissen eine Bedürfnislücke verursacht, die durch die unbewusst gelebte Phantasie einer erfolgreichen Inkorporation und Kontrolle zumindest temporär ausgefüllt werden kann. ${ }^{14}$ Insgesamt ist gleichwohl nicht davon auszugehen, dass orientalistische Erzeugnisse als Mittel zur Appropriation und Domestizierung mit Vorsatz von der Musikindustrie produziert werden, auch wenn etwaige Verschwörungstheorien z.B. im konkreten Bezug auf Hollywood immer wieder geäußert werden. ${ }^{15}$ Vielmehr darf angenommen werden, dass die

14 Dieses Phänomen ist von Nicolas Abraham und Maria Torok im Kapitel »Mourning or Melancholia: Introjection versus Incorporation« theoretisiert worden (vgl. Abraham/Torok 1995).

15 Ein prominenter Verfechter dieser Verschwörungstheorien ist der Gegen die Wand-Regisseur Fatih Akin. In einem Interview im Herbst 2006 gab er zu Protokoll: "I think that under Bush, Hollywood has been making certain films at the request of the Pentagon to normalise things like torture and Guantanamo. I'm convinced the Bush administration wants a third world war. I think they're fascists « (zit. n. Crossland 2006). 
Industrie schlicht dem orientalistischen musikindustrieimmanenten Diskurs zweiter Ebene folgt, solange er ökonomisch erfolgreich ist, d.h. von den Rezipienten angenommen wird. Wer sich in der westlichen Welt nicht völlig von allen Medien isoliert, ist täglich mit Orient/Okzident-Konfliktsituationen konfrontiert und hat damit potentiell ein unbewusst gelebtes Interesse an orientalistischen Kulturerzeugnissen; sie stellen ein Gegenwicht bereit gegen das Angstgefühl vor einer orientalisch-islamischen Bedrohung und zwar mittels einer auf die individuelle Psyche sedativ wirkenden Domestizierung und Inkorporation der vermeintlichen Kultur des Angst einflößenden Gegenübers.

Orientalistische Popprojekte als wechselseitige kulturelle Vermittler ansehen zu wollen, ist in den meisten Fällen nicht mehr als Wunschglaube oder schlichtes Marketingkalkül von Plattenfirmen zur Produktbewerbung. Sie sind auf unterschwellige Weise erfolgreich, weil sie hochaktuell und nahezu omnipräsent sind; sie sprechen eine sehr breite Zielgruppe durch alle audiovisuellen Medien an und bleiben durch tägliche Krisen und entsprechende Krisenberichterstattungen stets relevant und brisant.

Orientalistische Popmusik ist ein Paradoxon. Sie hat auf der einen Seite ein individuell sowie gesamtgesellschaftlich sedatives Potential; auf der anderen Seite reproduziert sie despektierliche und provokante Klischees, was das Okzident/Orient-Verhältnis belastet und nicht etwa entspannt, was wiederum weiteren Bedarf für neue orientalistische Kulturerzeugnisse mit impliziertem Beruhigungspotential schafft.

So gering die Aussichten im Jahre 2007 sein mögen, die Konfliktsituationen gänzlich bewältigen zu können, so groß ist die Wahrscheinlichkeit, dass der Orientalismus-Trend in der Popmusik noch viele Jahre überdauern wird und zwar einhergehend mit einer signifikanten und ästhetisch interessanten Ausdifferenzierung. Diese wird aller Voraussicht nach weiterhin aus westlichen Metropolen hervorgehen, die zum einen durch Migrationshintergründe von großen Teilen ihrer Bewohner ein hohes Maß an produktiver Interkulturalität versprechen und zum anderen stets mit Terrorgefahren konfrontiert sind, wie es die Beispiele New York und London in der jüngeren Vergangenheit gezeigt haben. 


\section{Literatur}

Abraham, Nicolas / Torok, Maria (1995). The Shell and the Kermel Vol. 1. Chicago, London: University Press.

Barthes, Roland (1964). Mythen des Alltags. Frankfurt/M.: Suhrkamp.

Browne, Nick (1989). »American Film Theory in the Silent Period: Orientalism as an Ideological Form. «In: Wide Angle 11, S. 23-31.

Berger, David / Peterson, Richard (1975). »Cycles in Symbolic Production: The Case of Popular Music. "In: American Sociological Review 40, S. 158-173.

Bernstein, Matthew / Studlar, Gaylyn (1997). "Introduction. «In: Visions of the East: Orientalism in Film. New Brunswick: University Press, S. 1-18.

Blades, James / Holland, James / Thrasher, Alan (2001). »Gong. «In: Grove Music Online. Hg. v. Laura Macy, http://www.grovemusic.com (Stand vom 26.3.2007).

Bloechl, Olivia (2005). »Orientalism and Hyperreality in >Desert Rose‘. In: Journal of Popular Music Studies 17, Nr. 2, S. 133-161.

Braddock, Kevin (2005). »Britney, Shakira and the Dawali Mania: Western Pop's New Orientalism. « In: http://www.kevinbraddock.com/features34.php (Stand vom 18.6.2007).

Crossland, David (2006). »Anti-Bush Statement Backfires in Germany: Film Director in Trouble Over Swastika T-Shirt.« In: Spiegel Online International vom 7. August, http://www.spiegel.de/international/0,1518,430542,00.html (Stand vom 26.3.2007).

Dyer, Richard (1992). »Don't Look Now: The Instabilities of the Male Pin-up.« In: Only Entertainment. Hg. v. Richard Dyer. New York: Routledge, S. 103-119.

Fanon, Frantz (1986). Black Skin, White Masks. London: Pluto.

Frith, Simon (1988). „Why do songs have words?« In: Ders., Music for Pleasure: Essays in the Sociology of Pop. Cambridge: University Press, S. 105-128.

Frith, Simon (2001). »The Popular Music Industry.«In: The Cambridge Companion to Pop and Rock. Hg. v. Simon Frith, Will Straw und John Street. Cambridge: University Press, S. 26-52.

Georgakas, Dan (1989). »The Arab Image in American Film and Television.« In: Cinéaste 17, Nr. 1, S. 1-24.

Hanna, Judith Lynne (1993). „Classical Indian Dance and Women's Status.« In: Dance, Gender and Culture. Hg. v. Helen Thomas. New York: Macmillan, S. 119135.

Leach, Elizabeth Eva (2001). »Vicars of >Wannabe: Authenticity and the Spice Girls.«In: Popular Music 20, Nr. 2, S. 143-167.

Lew, Alan A. / Hall, C. Michael / Williams, Allan M. (Hg.) (2004). A Companion to Tourism. Malden: Blackwell.

Locke, Ralph P. (1991). »Constructing the Oriental >Other<: Saint-Saëns's Samson et Dalila. «In: Cambridge Opera Journal 3, Nr. 3, S. 261-302.

Macfie, Alexander Lyon (Hg.) (2000). Orientalism: A Reader. New York: University Press.

McClary, Susan (Hg.) (1992). Georges Bizet: , Carmen . Cambridge: University Press. Mernissi, Fatima (2001). Scheherazade Goes West. Washington: Square Press.

Michalek, Laurence (1989). "The Arab in American Cinema: A Century of Otherness. « In: Cinéaste 17, Nr. 1, S. 3-9. 
Ott, Clara (2006). »Wieso sind Konzerte so teuer? [Interview mit Peter Cadera].« In: ZEIT online vom 7. Februar, http://nurtext.zeit.de/online/2006/06/cadera (Stand vom 26.3.2007).

Parfitt, Clare (2005). »The Spectator's Dancing Gaze in Moulin Rouge! «In: Research in Dance Education 6, Nr. 1/2, S. 97-110.

Robinson, Peter (1993). »Is Aida an Orientalist Opera?« In: Cambridge Opera Journal 5, Nr. 2, S. 133-140.

Said, Edward (1985). »Orientalism Reconsidered.«In: Cultural Critique 1, S. 89107.

Said, Edward (2003). Orientalism. New York: Vintage.

Scott, Derek B. (1998). »Orientalism and Musical Style.«In: Musical Quarterly 82, Nr. 2, S. 308-335.

Shaheen, Jack G. (1987). »The Hollywood Arab (1984-1986). «In: Journal of Popular Film and Television 14, Nr. 4, S. 148-157.

\title{
Tonträger und Videos:
}

Pussycat Dolls (2006a). »Buttons«. Auf: CD-Single Buttons. A\&M Records, ASIN: B000G004T2 (CD enthält Video).

Pussycat Dolls (2006b). »Buttons« [Video]. In: http://www.youtube.com/results? search_query=buttons+pussycat+dolls (Stand vom 26.3.2007).

Maroon 5 (2002). "Shiver«. Auf: Songs About Jane. J Records, ASIN: B000094BZ5.

\begin{abstract}
This article problematises a contemporary phenomenon which has become significantly apparent since the turn of the millennium: The incorporation of putative Oriental music elements in numerous pop songs released by transnationally operating labels of the Western music industry. For this purpose, Edward Said's seminal study Orientalism is applied as a methodical framework and source for critical theses. The paper debates urban conditions, music industry discourses as well as extra-musical factors that have been essential for the origination of the Orientalism trend. The main part, however, concentrates on the detailed analysis of two examples of Orientalism in popular music: the Pussycat Dolls' »Buttons « and Maroon 5's »Shiver «. The paper discusses the correlations between the omnipresent cultural and political Oriental/Occidental crisis and the evident success of Orientalist pop pieces.
\end{abstract}

